\title{
Unsharp Localization and Causality in Relativistic Quantum Theory
}

\author{
Paul Busch \\ Former address: Department of Mathematics, The University of Hull, Hull, UK \\ Current address: Department of Mathematics, University of York, York, UK \\ Electronic mail: paul.busch@york.ac.uk
}

Published in: J. Phys. A: Math. Gen. 32 (1999) 6535-6546

DOI: $10.1088 / 0305-4470 / 32 / 37 / 305$

\begin{abstract}
The conflict between relativistic causality and localizability is analyzed in the light of the existence of unsharp localization observables. A theorem due to S. Schlieder is generalized, showing that the assumption of local commutativity implies the localization observable in question to be unsharp in a strong sense. Furthermore, a recent generalization of a theorem of Lüders is applied to demonstrate that local commutativity is a necessary consequence of Einstein causality even in the case of unsharp observables if they admit local measurements. These findings raise the question whether localization observables can be measured by means of local operations.
\end{abstract}

\section{Introduction}

The concept of localization raises intriguing problems in relativistic quantum theory. On one hand, the idea of localizability (of particles, centres of charge or energy distributions, etc.) has always had an unquestionable heuristic and interpretational value. On the other hand, any attempt at a formalization of localization as an observable seems to face a fundamental conflict with the requirement of causality on which (together with some other postulates) relativistic quantum theories are built. This conflict is epitomised in theorems due to S. Schlieder 11 and G. Hegerfeldt 2].

Further problems arise in the context of the relativistic quantum mechanics of (free) particles. For example, it has been noted that a conserved probability current with positive probability density does not exist in all cases (e.g., 3. 4). Furthermore, no sharp localization observable exists for particles with zero mass and spin at least one 5 . These problems are overcome in an approach that describes (spatial) localization in terms of marginal observables of 
covariant phase space observables (e.g., [6, 7, 9]). Such observables are unsharp observables represented as noncommutative positive operator measures (POM's), thus accounting for the noncommutativity of position and momentum and the uncertainty relation. This success raises the question whether localizability and causality can be reconciled for unsharp (spatial) localization observables. A pragmatic answer ("FAPP") has been given in the phase space approach in [8], indicating that the possibility for causality violating behavior is spurious.

In the present paper Schlieder's theorem is reconsidered in order to decide whether its statement also holds in the case of unsharp observables. It turns out that localization observables will necessarily be unsharp in a strong sense if they are to satisfy the local commutativity condition (Section 2). The question of whether the local commutativity of unsharp localization observables is in turn a necessary consequence of Einstein causality is addressed in Section 3. General conclusions and problems for future investigation are summarized in Section 4. For the readers' convenience, basic concepts relating to unsharp observables represented as POM's are briefly reviewed in Appendix 1.

\section{Unsharp Localization vs. Local Commutativ- ity}

Schlieder's theorem [1] is based on the following structures commonly accepted as fundamental for any relativistic quantum theory.

(a) a complex Hilbert space $\mathcal{H}$, the rays of which represent pure states of the system;

(b) a strongly continuous unitary representation $a \mapsto U(a)$ in $\mathcal{H}$ of the translation group of Minkowski space $M$;

(c) for any future directed, timelike unit vector $a$, the generator $H(a)$ (Hamiltonian) is bounded below (spectrum condition).

Within the structure $(\mathcal{H}, a \mapsto U(a))$, the following conditions are taken to characterize a (spatial) localization observable within a given inertial frame.

(0) Localization Event Structure: Fix a foliation of Minkowski space $M$ by means of a family $\mathcal{S}$ of parallel spacelike hyperplanes $S$; each $S$ is required to be equipped with a family $\mathcal{F}(S)$ of subsets, called spatial sets, including a (covering) family of bounded subsets, and such that $\mathcal{F}(S+a)$ consists of the translates by $a$ of the sets from $\mathcal{F}(S)$; and a map $\Delta \mapsto E_{\Delta}$ from $\mathcal{F}(S)$ to effects of $\mathcal{H}$ for each $S$.

(1) Translation Covariance: For all $a \in M$,

$$
U(a) E_{\Delta} U(a)^{*}=E_{\Delta+a} .
$$


(2) Localizability: For each $S \in \mathcal{S}$ and $\Delta_{1}, \Delta_{2} \in \mathcal{F}(S)$,

$$
\text { if } \Delta_{1} \cap \Delta_{2}=\emptyset \text { then } E_{\Delta_{1}} E_{\Delta_{2}}=E_{\Delta_{2}} E_{\Delta_{1}}=0 \text {. }
$$

(3) Local Commutativity: For $S_{1}, S_{2} \in \mathcal{S}, \Delta_{1} \in \mathcal{F}\left(S_{1}\right), \Delta_{2} \in \mathcal{F}\left(S_{2}\right)$,

$$
\text { if } \Delta_{1}, \Delta_{2} \text { spacelike separated then } E_{\Delta_{1}} E_{\Delta_{2}}=E_{\Delta_{2}} E_{\Delta_{1}} \text {. }
$$

The theorem then reads:

Theorem 1 If the structure $\left(\mathcal{H}, a \mapsto U(a), \Delta \mapsto E_{\Delta}\right)$, with the $E_{\Delta}$ being projections $P_{\Delta}$, satisfies conditions (1)-(3), then $P_{\Delta}=0$ for all bounded spatial sets $\Delta$.

The present formulation of the theorem for projection valued maps $\Delta \mapsto P_{\Delta}$ is due to D. Malament [10]. In Schlieder's original version, condition (3) was replaced with the somewhat stronger requirement (referred to as a consequence of causality and similar to Hegerfeldt's [2] characterization of causality) that the product $P_{\Delta_{1}} P_{\Delta_{2}}=0$ for spacelike separated pairs of sets; then it is pointed out that the localization projections $P_{\Delta}$ cannot belong to any local algebra. Examples of such 'nonlocal' localization observables are given by the Newton-Wigner position operators [11] or the corresponding localization spectral measures constructed by Wightman [5]. It should be noted that these sharp localization observables do satisfy the covariance condition (1) [12.

Theorem 1 has been interpreted as implying the impossibility of a physically acceptable notion of localizability of physical systems in relativistic quantum theories. Inasmuch as a localization observable is a defining feature of a particle, this conclusion would entail the impossibility of a relativistic quantum mechanics of particles. However, before subscribing to such far-reaching conclusions, it is worthwhile to reanalyze the assumptions in some detail in view of the possibility that localization might be an intrinsically unsharp observable.

In what follows we will not question the postulates (a), (b) and (c), nor the property (1) of translation covariance. We will also tentatively adopt the view that the operational definition of spatial sets and the continuum of possible spacetime translations can be realized with arbitrary accuracy with classical physical, macroscopic means. The implications of the fact that measuring devices such as detectors are composed of quantum constituents will only be explored in the discussion at the end.

The formulation of the localization event structure is usually somewhat sharpened by requiring the maps $\Delta \mapsto E_{\Delta}$ to be POM's defined on spatial Borel sets. As we shall see, this would simplify some of the arguments below. Physically, it would reflect the assumption that localization is operationally meaningful for arbitrarily small sets, which could again be challenged on the grounds that detectors are composed of quantum systems.

First we investigate the question whether or not the statement of Theorem 1 extends to unsharp localization observables as well. To this end we will allow the structure $\left(\mathcal{H}, a \mapsto U(a), \Delta \mapsto E_{\Delta}\right)$ to be based on effects $E_{\Delta}$ which are not necessarily projections. 
Theorem 2 If the structure $\left(\mathcal{H}, a \mapsto U(a), \Delta \mapsto E_{\Delta}\right)$ satisfies conditions (1)(3), then $E_{\Delta}=0$ for all (bounded) spatial sets $\Delta$.

We will sketch a proof of this statement as it is convenient to display the technicalities involved - they are exactly the same as in the original case. As in that case, the proof rests on the following substantial result due to Borchers [13, presented here in terms of effects rather than projections only.

Lemma 1 Let $V(t)$ be a strongly continuous one-parameter group of unitary operators on a Hilbert space whose generator $H$ has a spectrum bounded from below. Let $E_{1}, E_{2}$ be two effects such that

(i) $E_{1} E_{2}=0$, and

(ii) there is $\varepsilon>0$ such that for all $t$ with $|t|<\varepsilon,\left[E_{1}, V(t) E_{2} V(t)^{*}\right]=0$.

Then $E_{1} V(t) E_{2} V(t)^{*}=0$ for all $t \in \mathbb{R}$.

Proof. We only show how the statement can be reduced to the known one for projections. Let $P_{1}, P_{2}$ be the projections onto the ranges of $E_{1}, E_{2}$, respectively. Then $P_{1}^{(0)}=I-P_{1}, P_{2}^{(0)}=I-P_{2}$ are the projections onto the kernels. Now, observe that $E_{1} E_{2}=0 \Leftrightarrow P_{1} P_{2}=0$. [In fact: $E_{1} E_{2} \varphi=0 \forall \varphi \Leftrightarrow$ $\operatorname{ran}\left(E_{2}\right) \subseteq \operatorname{ker}\left(E_{1}\right) \Leftrightarrow P_{2} \leq P_{1}^{(0)} \Leftrightarrow P_{2}\left(I-P_{1}\right)=P_{2} \Leftrightarrow P_{2} P_{1}=0$.] Next observe that $V(t) P_{2} V(t)^{*}$ is the projection onto the range of $V(t) E_{2} V(t)^{*}$. Hence, (ii) implies $\left[P_{1}, V(t) P_{2} V(t)^{*}\right]=0$ for all $t$ with $|t|<\varepsilon$. So we have (i) and (ii) for $P_{1}, P_{2}$, and therefore $P_{1} V(t) P_{2} V(t)^{*}=0$ for all $t$. But this is equivalent to $E_{1} V(t) E_{2} V(t)^{*}=0$ for all $t$.

It is straightforward to check that Malament's line of argument goes through for effects $E_{\Delta}$ in place of projections $P_{\Delta}$, yielding $E_{\Delta}=0$ for all bounded $\Delta$. We will not carry this out here but later in a somewhat less trivial context. Here we note the following. In view of the probabilistic interpretation of the operators $E_{\Delta}$, if $\Delta_{1}, \Delta_{2} \in \mathcal{F}(S)$ are two disjoint subsets, then the sum of expectations of $E_{\Delta_{1}}$ and $E_{\Delta_{2}}$ should represent the probability of localizing the system in $\Delta_{1} \cup$ $\Delta_{2}$, and the operator representing these probabilities is $E_{\Delta_{1} \cup \Delta_{2}}$. Thus one is led to stipulate the additivity of the map $\Delta \mapsto E_{\Delta}$, that is, $E_{\Delta_{1}}+E_{\Delta_{2}}=E_{\Delta_{1} \cup \Delta_{2}}$ for disjoint $\Delta_{1}, \Delta_{2} \in \mathcal{F}(S)$. In addition, one might consider the possibility that localization somewhere in a given hyperplane would occur with certainty, that is, $E_{S}=I$. We note that for these probabilistic requirements to be implemented, it is necessary that the family $\mathcal{F}(S)$ is an algebra. [One usually assumes it to be a a $\sigma$-algebra, such as, for instance, the Borel algebra of $S$.] But then for a normalized POM, the localization condition (2) is seen to be equivalent to the effects $E_{\Delta}$ being projections. In fact (2) implies $E_{\Delta}\left(I-E_{\Delta}\right)=0$, that is, $E_{\Delta}^{2}=E_{\Delta}$. The converse implication is trivial. So if localization observables were adequately represented as normalized POM's, the contents of Theorem 2 would immediately reduce to that of the original theorem. But one should keep in mind that the normalization condition does not apply to all POM's representing physical observables.

Our main criticism of Theorem 2 aims at another aspect: the formalization of the localization condition in terms of the algebraic condition $E_{\Delta_{1}} E_{\Delta_{2}}=0$ for 
disjoint spatial sets. What one actually tries to express with this condition is the following: "If the system is in $\Delta_{1}$, it certainly is not in $\Delta_{2}$ whenever these sets are disjoint." Thus, (2) should be replaced with

(2') For all states $\varphi \in \mathcal{H},\|\varphi\|=1, \Delta_{1}, \Delta_{2} \in \mathcal{F}(S)$,

$$
\text { if } \Delta_{1} \cap \Delta_{2}=\emptyset \quad \text { then }\left\langle\varphi \mid E_{\Delta_{1}} \varphi\right\rangle=1 \Longrightarrow\left\langle\varphi \mid E_{\Delta_{2}} \varphi\right\rangle=0 \text {. }
$$

This is equivalent to $P_{\Delta_{1}}^{(1)} \leq P_{\Delta_{2}}^{(0)}$, where $P_{\Delta}^{(1)}, P_{\Delta}^{(0)}$ denote the spectral projections of $E_{\Delta}$ associated with the eigenvalues 1 and 0 , respectively. It is obvious that this condition is equivalent to (2) in the case of projections. For effects, (2') only implies $P_{\Delta_{1}}^{(1)} P_{\Delta_{2}}^{(1)}=0$ while in general $E_{\Delta_{1}} E_{\Delta_{2}} \neq 0$. Note that (2') can be obtained as a consequence of the assumption that $\Delta \mapsto E_{\Delta}$ is a (not necessarily normalized) POM: if, for disjoint $\Delta_{1}, \Delta_{2}, E_{\Delta_{1}}+E_{\Delta_{2}}=E_{\Delta_{1} \cup \Delta_{2}}(\leq I)$ and $\left\langle\varphi \mid E_{\Delta_{1}} \varphi\right\rangle=1$, then $\left\langle\varphi \mid E_{\Delta_{2}} \varphi\right\rangle=0$.

Theorem 3 If the structure $\left(\mathcal{H}, a \mapsto U(a), \Delta \mapsto E_{\Delta}\right)$ satisfies conditions (1), (2') and (3), then $P_{\Delta}^{(1)}=0$ for all (bounded) spatial sets $\Delta$.

In Appendix 2 we show how to adjust the proof of Theorem 1 to obtain this result. We interpret this result as follows: if there exists a localization observable satisfying the conditions of Theorem 3 then this observable is necessarily strongly unsharp in the sense that its effects $E_{\Delta}$ do not have eigenvalue 1 . This leaves us with the question whether among the strongly unsharp, covariant localization observables there exist any that satisfy local commutativity. To my knowledge the answer is not known. An indication to the negative is provided by a recent theorem stating that spacetime localization observables cannot belong to any quasilocal algebra 14 .

It is known that among the relativistic phase space observables there are strongly unsharp observables, whose spatial marginals would thus in general be strongly unsharp covariant localization observables. In the remaining part of the paper we address the question of the relevance of the local commutativity condition (3) in the case of unsharp localization observables: is it a necessary consequence of the requirement of Einstein causality? We shall find a partial answer and along the way obtain an indication that phase space localization observables do violate local commutativity.

\section{Local Commutativity and Causality for Un- sharp Localization Observables}

The term "Einstein causality" refers to the intuitive idea of physical processes propagating with at most the velocity of light. Following Schlieder [1] and Hegerfeldt [2, we will distinguish between weak and strong (Einstein) causality: the former refers to subluminal propagation of changes of expectation values, whereas the latter describes subluminal propagation of individual, definite properties. Alternative terms in use are macro- and microcausality, respectively. 
For sharp observables, the local commutativity condition is known to be equivalent to weak Einstein causality [15. This result is a consequence of a famous theorem due to Lüders [16] which states the following: two (discrete) observables represented by self-adjoint operators $A, B$ commute if and only if for any state, the statistics of a measurement of $B$ is not affected by a nonselective Lüders measurement of $A$ (that is, a measurement without reading). This theorem can be extended to observables whose spectra are not discrete: the commututativity $A, B$ is equivalent to the statistical nondisturbance condition being stipulated for Lüders measurements on all discrete coarse-grainings of the observable $A$. Now let $A, B$ be observables that can be measured in two spacelike separated regions of spacetime, respectively. Then weak Einstein causality requires that acts of measurements in one region should not have statistically significant effects in another region at a spacelike separation. This is captured by the nondisturbance statement in the Lüders theorem.

There are strong indications that the Lüders theorem extends to the case of unsharp observables: in fact two important special cases have been proven recently [17. It can be argued that these two cases are sufficiently comprehensive for physical purposes. They will serve the present needs. The formulation of the following proposition rests on the notion of a Lüders measurement for unsharp observables. Let $A$ be an unsharp observable represented by a complete family of effects, $A=\left\{E_{i}\right\}_{i=1 \ldots N}, \sum_{i} E_{i}=I$. A nonselective Lüders measurement of $A$ leads to a state change of the object that is described by the Lüders state transformation, defined via

$$
\rho \longmapsto \mathcal{I}_{L}^{A}(\rho):=\sum_{i=1}^{N} E_{i}^{1 / 2} \rho E_{i}^{1 / 2}
$$

for all state operators $\rho$ (for details on this concept, cf. [18). One considers the question under what conditions the outcome of a measurement of an effect $B$ does not depend on whether or not a nonselective measurement of $A$ has been carried out.

Proposition 1 Let $A=\left\{E_{i}\right\}_{i=1 \ldots N}$ be a collection of effects such that $\sum_{i} E_{i}=$ $I$, and let $B$ be an effect. Then the equivalence

$$
\left[B, E_{i}\right]=0 \quad \forall i \quad \Longleftrightarrow \quad \operatorname{tr}[\rho B]=\operatorname{tr}\left[\mathcal{I}_{L}^{A}(\rho) B\right] \quad \forall \rho
$$

holds (at least) in the following two cases:

( $\alpha$ ) $B$ has a discrete spectrum of eigenvalues that can be ordered in decreasing order, $A$ arbitrary;

(B) $A=\left\{E_{1}, I-E_{1}\right\}, B$ arbitrary.

In order to formulate weak Einstein causality, we need to assume a physically meaningful association of observables with (bounded open) spacetime regions, in the sense that such observables can be measured by means of operations carried out within these regions. Such measurements, operations and observables will 
be called local. In the context of algebraic relativistic quantum theory, a measurement is local if the operations representing the associated state changes are expressible in terms of elements of the corresponding local algebra of observables [19. This condition is satisfied for Lüders measurements. Now, weak Einstein causality means the following: if $A$ and $B$ represent observables associated with two spacelike separated spacetime regions $R_{1}, R_{2}$, respectively, then the act of a nonselective local measurement of $A$ should not influence the outcomes of a measurement of $B$ (and vice versa). Hence, weak Einstein causality for Lüders measurements of local observables reads:

$$
R_{1}, R_{2} \text { spacelike separated } \Longrightarrow \operatorname{tr}[\rho B]=\operatorname{tr}\left[\mathcal{I}_{L}^{A}(\rho) B\right] \forall \rho .
$$

The state description $\mathcal{I}_{L}^{A}(\rho)$ is appropriate to local observers acting in $R_{2}$ since - given that there are no classical signals faster than light - it represents the information available to them if they know that a measurement in $R_{1}$ is taking place. In view of Proposition 1, it follows that for local measurements of unsharp observables in spacelike separated regions to satisfy weak Einstein causality, these observables must commute. This is remarkable as local measurements at spacelike separations can be regarded in a way as joint measurements, and it is known that unsharp observables can be jointly measurable without necessarily commuting with each other.

Schlieder's argument can now be formulated for localization observables as follows.

Proposition 2 Let $A=\left\{E_{1}, E_{2}\right\}$, where $E_{1}=E_{\Delta_{1}}, E_{2}=I-E_{\Delta_{1}}$ and $B=$ $E_{\Delta_{2}}$ are localization effects, and $\Delta_{1} \in \mathcal{F}\left(S_{1}\right), \Delta_{2} \in \mathcal{F}\left(S_{2}\right)$ are bounded spatial sets contained in spacelike separated regions $R_{1}, R_{2}$, respectively. Suppose that $A$ and $B$ are locally measurable in these regions and that the Einstein causality condition $(C)$ holds for them. Then $E_{\Delta_{1}}$ and $E_{\Delta_{2}}$ commute.

This statement is an immediate consequence of case $(\beta)$ of Proposition 1 It is interesting to observe the following consequence: if a localization observable $\Delta \mapsto E_{\Delta}$ is represented as a POM and measurable by means of local operations, then causality requires this POM to be commutative since all bounded disjoint spatial sets of the $\sigma$-algebra $\mathcal{F}(S)$ are spacelike separated and hence commute with each other. On the other hand, covariant relativistic phase space observables are constructed via generalized coherent states and thus are noncommutative (see [6, 7]). Hence, such phase space observables cannot satisfy local commutativity. This is not too surprising as a phase space localization measurement involves a measurement of momentum which itself is not a local observable. However, there is a possibility that the spatial marginals of a suitable covariant family of phase space observables are commutative.

Besides weak causality, there is another, independent requirement that entails local commutativity. This is the condition of (relativistic) objectivity: the descriptions of a pair of spacelike separated local measurements given by different inertial observers should be consistent with each other. Thus, consider 
two inertial observers in the intersection of the forward lightcones (causal influence regions) of the two spacelike separated regions $R_{1}, R_{2}$. Suppose the observers are moving relative to each other in such a way that they assign different time orderings to the two local measurements. Relativistic objectivity means that they predict and record the same statistics for all possible future measurements. Therefore, although the time orderings are different, the successive state changes should nevertheless lead to the same final state in both descriptions. Let $A=\left\{E_{1}, E_{2}, \ldots\right\}, \sum E_{i}=I$, and $B=\left\{F_{1}, F_{2}, \ldots\right\}, \sum F_{j}=I$ represent the two discrete local observables in question, and let $\mathcal{I}_{L, i}^{A}, \mathcal{I}_{L, j}^{B}$ denote the associated Lüders operations. Then objectivity is expressed by the following condition [15]:

$$
\begin{aligned}
R_{1}, R_{2} \text { spacelike separated } \Longrightarrow & \mathcal{I}_{L, i}^{A}\left(\mathcal{I}_{L, j}^{B}(\rho)\right)=\mathcal{I}_{L, j}^{B}\left(\mathcal{I}_{L, i}^{A}(\rho)\right) \\
& \text { for all } \rho, i, j .
\end{aligned}
$$

It can be shown that the commutativity of the Lüders operations for $A$ and $B$ is equivalent to the commutativity of all $E_{i}$ with all $F_{j}$ [20]. (For pairs of observables in the domain of applicability of Proposition 1, this equivalence is a consequence of the fact that due to $\sum_{i} \mathcal{I}_{L, i}^{A}=\mathcal{I}_{L}^{A}$, the commutativity property in $(\mathrm{O})$ implies the nondisturbance property in $(\mathrm{C})$ ). Therefore, the relativistic objectivity condition is equivalent, via local commutativity, to weak Einstein causality. This important connection (e.g., [21] and references therein) is thus found to be valid also for unsharp observables. The fact that the class of measurements used in these arguments is of the Lüders type is only of technical significance: weak causality and objectivity would be necessary requirements to be imposed on all local measurements and therefore, in particular, on Lüders measurements.

The potential conflict between causality and localizability for relativistic quantum mechanics has been highlighted by Hegerfeldt on the basis of a notion of strong causality that requires subluminal propagation of localization properties (for a concise review, see [2]). More precisely, strong causality requires that for a bounded spatial set $\Delta$, if the probability of localization at time $t=0$ is 1 , then the probability of localization at time $t>0$ within the inflated set $\Delta_{t}=\{x \in S \mid \operatorname{dist}(x, \Delta) \leq c t\}$ is also equal to 1 . It is shown that this condition cannot be satisfied by any (covariant) localization observable.

It is not hard to see that in the case of sharp localization observables represented as a normalized projection valued measure $\Delta \mapsto P_{\Delta}$, the strong causality implies local commutativity (3) and thus weak causality. Translating this into the Heisenberg picture, this means $P_{\Delta} \leq P_{\Delta_{t}+t a}$, where $a$ is the future timelike unit vector perpendicular to $S$. Now let $\Delta_{1} \in S, \Delta_{2} \in$ $S+$ ta be two spacelike separated bounded spatial sets. Strong causality yields $P_{\Delta_{1}} \leq P_{\Delta_{1, t}+t a} \leq I-P_{\Delta_{2}}$, and so $P_{\Delta_{1}} P_{\Delta_{2}}=0$. Thus, the violation of local commutativity for sharp localization observables entails the violation of strong causality. It should be noted, however, that Hegerfeldt's theorem takes into account the possibility of localization operators $E_{\Delta}$ which are effects but not projections. In this case strong causality implies the following chain of inclusions 
for the respective spectral projections:

$$
P_{\Delta_{1}}^{(1)} \leq P_{\Delta_{1, t}+t a}^{(1)} \leq P_{\Delta_{2}}^{(0)} \leq I-P_{\Delta_{2}}^{(1)},
$$

and so $P_{\Delta_{1}}^{(1)} P_{\Delta_{2}}^{(1)}=0$. It is not clear whether in this case strong causality does imply weak causality (for localization effects). But the assumption of local commutativity, via Theorem 3, is seen to render strong causality inapplicable: the premise of strict initial localization cannot be fulfilled. Therefore, in the present framework strong causality is an unnecessary assumption. Its virtue lies in the fact that some of the other postulates (such as covariance and even the group representation) could be dropped and still strong causality is violated in the sense of instantaneous delocalization of wave functions 2]. However, without translation covariance - which we regard as a defining property of localization observables - it appears doubtful whether such delocalizations can be interpreted as giving rise to (possible) superluminal particle propagations.

\section{Discussion}

We have generalized a theorem due to Schlieder which states the incompatibility between local commutativity and sharp covariant localizability. Based on a generalization of Lüders' theorem, we then found that local commutativity is equivalent to weak Einstein causality as well as to a postulate of relativistic objectivity, also in the case of unsharp observables - provided they admit local measurements. The results of Sections 2 and 3 leave us with the following situation.

(I.) For localization observables $\Delta \mapsto E_{\Delta}$ admitting sharply localized states (i.e., states which yield probability 1 in bounded spatial sets), local commutativity is violated (Theorems 1 and 3). If there is any physical sense in saying that the effects $E_{\Delta}$ are measurable by local operations, then weak Einstein causality would be violated (Propositions 1 and 2). Such local, sharp localization measurements would lead to statistical influences between spacelike separated regions and hence, superluminal signals.

(II.) For localization observables to satisfy local commutativity, they must necessarily be strongly unsharp. It is an open question whether local commutativity is actually satisfied for any or all strongly unsharp localization observable; an interesting (though unlikely) class of candidates to be investigated is given by the spatial marginals of phase space observables. If a strongly unsharp localization observable can be measured by local operations, and if it violates local commutativity, this would again imply a violation of weak Einstein causality.

The problematic conclusion in (I.) can be countered by arguing that sharp spatial localization is an operationally meaningless idealization. It would in fact seem implausible to ignore the quantum nature of the constituents of the detectors used to define spatial localization sets. It appears more likely that realistic procedures for measuring localization observables, which are based on quantum probes with extended wave functions and interactions with infinite 
ranges (albeit with decreasing strengths), would render the spatial localization sets intrinsically fuzzy. Also, confining a quantum object or probe within sharp spatial boundaries would require an infinite amount of energy, (e.g., an infinite potential well). Thus, the causality violation for sharp localizations could be seen as an artefact arising from an unjustified idealization. (From the perspective of a relativistic quantum field theory, such strong interactions as would be needed for sharp localization measurements would give rise to particle pair creations and could not therefore be described within a single-particle theory. However, we would prefer a decision on the limitations of a single-particle relativistic quantum mechanics from within that theory.)

The conclusion of causality violations for either sharp localizations (scenario (I.)) or strongly unsharp localizations violating local commutativity (scenario (II.)) may be barred for another, common reason: localization observables could simply fail to be local observables. In fact, the concept of a localization observable - whether sharp or unsharp - involves global elements, namely, the totality of all bounded spatial subsets of $S$ as well as the defining requirement of translation covariance. It is thus quite conceivable that from their very operational definition, sharp or unsharp localization effects cannot be regarded as locally measurable quantities. As local measurability is a premise of the weak Einstein causality postulate (and of the objectivity requirement), this postulate and, along with it, Proposition 2 would in this situation become inapplicable. Thereby the validity of weak causality would not be affected by a violation of the commutativity condition (3); indeed this condition would lose its intended meaning indicated by the phrase 'local commutativity'. This interpretation of Theorems 1 and 3 is in accordance with the analysis given by Schlieder [1: in the context of a relativistic local quantum (field) theory, which stipulates local commutativity, Theorem 1 is interpreted as implying that localization observables admitting sharp localization cannot belong to a local algebra.

The assumption of local measurability of localization observables has also been challenged by Butterfield and Fleming [22] in a recent lucid analysis of the 'strange' properties of localizations. I feel this point requires a detailed measurement theoretic investigation before Schlieder's theorem could be taken as conclusive evidence against the possibility of a relativistic quantum mechanics of particles in the sense proposed by Malament [10].

If it is granted that localization observables allowing sharply localized states are not local observables, so that weak causality would not be challenged, then Hegerfeldt's theorem would still entail the instantaneous spreading of wave functions and hence potential superluminal propagation of a particle. I would argue that this cannot be used for superluminal signalling: In order for the particle to carry a bit of information, the sender would need to be able to control the particle to the extent that (s)he is capable of either releasing the particle or keeping it trapped. This would entail the infinite energy problem mentioned above. On the other hand, if the particle is free, the 'sender' will have no control over it, and the fact that it had been localized in a bounded region at some initial instant of time does not by itself carry any information to a spacelike separated receiver. 
The preceding lines of reasoning can be carried somewhat further. Up to now we have assumed that bounded spacetime regions - in which local physical operations are to be carried out - can be operationally defined solely by classical physical means. Thus it is assumed that the quantum nature of the constituents of the relevant measuring devices can be ignored. It would be the task of a relativistic quantum theory of spacetime measurements, which remains yet to be developed, to justify this assumption. As indicated above, sharp localization may not be an operationally meaningful concept; given that the (quantum) devices used to define spacetime regions are themselves only unsharply localizable, the concept of a local measurement - and with it that of a local observable algebra - would have to be reformulated. This would render weak and strong Einstein locality inapplicable and would call for an operationally significant notion of causality, possibly in the form of a probabilistic concept and involving reference to appropriate levels of detector sensitivities.

Hegerfeldt has proposed a probabilistic causality condition according to which the 'bulk' of a wave packet propagates with subluminal speed. This condition is still found to be violated for approximately localized states with fast decaying ('exponential') tails [2]. On the other hand, it has been shown that the probabilities for causality violating behavior are spurious from a practical point of view (e.g., [8]). However, the experimental relevance of the present formulations of probabilistic causality does not seem entirely evident; in particular, it is again unclear whether violations of this causality condition would permit the existence of superluminal signals.

Finally we point out that a coherent account of the status of postulates such as local commutativity, or weak and strong causality in current relativistic quantum theories is needed not only for the sake of theoretical argument. In recent years, various experimental groups have reported demonstrations of superluminal propagation phenomena with evanescent microwave modes and with pairs of photons passing through opaque media. An up to date discussion of such experiments can be found in the special Proceedings issue of Annalen der Physics cited in Refs. [2, 21]. Some authors seem close to suggesting that the possibility of superluminal signalling on the basis of such experiments may not be ruled out. Such a claim cannot be refuted simply by making reference to the fact that our existing relativistic quantum theories incorporate the principle of weak causality: they do so because it has been built in 'by hand', namely by stipulation of local commutativity. This situation raises the interesting question as to what could constitute a principal theoretical demonstration of the impossibility of superluminal signals. (It appears to me that it would be hard to dismiss relativistic objectivity, which seems to constitute a necessary precondition for any scientific theory; and as we have seen, within Hilbert space quantum theory this implies local commutativity and weak causality.) As long as a plain experimental demonstration of superluminal signals is lacking (which I suspect it will be for a very long time), the compatibility of these experiments with relativistic causality could only be demonstrated by way of providing a satisfactory quantitative account of the experiments using relativistic quantum theory; this would amount to giving a causal explanation. Such a study will 
have to be based on the use of sound localization observables for the photons involved; and these are known to be necessarily strongly unsharp [9. Thus, all the questions raised above concerning the (non-)locality and possible probabilistic causality of localizations will have to be addressed. Some of these issues will be taken up in a forthcoming study by J. Brooke and the author [23].

\section{Acknowledgement}

It is a pleasure to thank James Brooke for helpful comments on a previous version of the manuscript.

\section{Appendix 1: Effects, POM's, Unsharpness, and All That}

Operational Quantum Theory is a conceptual completion of quantum mechanics on Hilbert space $(\mathcal{H})$ in the sense that the most general notion of observable is incorporated that is compatible with the probabilistic structure of the theory. An observable encompasses the totality of statistics of a given measurement (or class of measurements yielding the same statistics) with respect to all input states. Insofar as the probability for an outcome is to be given in terms of the expectation value of some operator in any state, it follows that such an operator must be positive and have a spectrum within the interval $[0,1]$. Operators of this kind, which represent the occurrence of a particular outcome in a measurement, are called effects. Hence, a linear operator $E$ is an effect if it is bounded by $O$ and $I, O \leq E \leq I$. An observable will thus be constituted by an association of effects with subsets of possible values. The probability for an outcome in one of a collection of disjoint value sets should be given by the sum of probabilities for the separate sets. This leads to the additivity property of the map from value sets to effects. For convenience, one allows additivity for finite or countably many disjoint sets and refers to this as $\sigma$-additivity. In addition, it is usually assumed that for a given measurement it is certain that some outcome will occur, that is, that the probability for the total value set, $\Omega$, is equal to unity. This is to say that the associated effect in the unit operator, $I$. One is thus led to the definition of an observable as a normalized positive operator valued (or effect valued) measure $\Sigma \ni X \longmapsto E(X)$ defined on a measurable space $(\Omega, \Sigma)$ such that:

$$
\begin{aligned}
O & \leq E(X) & & \text { (positivity) } \\
E\left(\bigcup_{i} X_{i}\right) & =\sum_{i} E\left(X_{i}\right) \quad \text { if } X_{j} \cap X_{k}=\emptyset, j \neq k & & \text { ( } \sigma \text {-additivity) } \\
E(\Omega) & =I & & \text { (normalization) }
\end{aligned}
$$

It should be noted that observables in the usual sense are captured by this definition by virtue of the spectral theorem: first of all, the set of effects contains 
all projections; and any self-adjoint operator gives rise to a unique projection valued measure on the real Borel algebra $(\mathcal{B}(\mathbb{R}))$, its spectral measure. Effect valued measures that are not projection valued are distinguished from projection valued measures by the following important property: a projection $P$ and its complement, $P^{\perp}:=I-P$, are necessarily orthogonal to each other, that is, $P P^{\perp}=O$. Conversely, if an effect $E$ and its complement $E^{\perp}:=I-E$ satisfy $E E^{\perp}=O$ then $E$ is a projection (as $E=E^{2}$ ). This fact suggests the definition of a sharp observable as an effect valued measure such that for any effect $E$ in its range, $E$ and its complement $E^{\perp}$ have no common positive, nonzero lower bound; this is equivalent to $E E^{\perp}=O$, and hence to the statement that the effect valued measure is actually a projection valued measure. All other observables will be referred to as unsharp observables. Note that it is possible for an unsharp observable to have definite values, namely, if the effect associated with some value or range of values has eigenvalue 1 . The term unsharpness only refers to the fact that some effects in the range of the observable have a spectrum within $[0,1]$ not limited to (a subset of) $\{0,1\}$. For further information on unsharp observables, the reader may refer to the monograph [18].

\section{Appendix 2: Proof of Theorem 3}

We follow Malament (1996) step by step, the only modification being an appropriate choice of the pairs of projections in question. The reader may wish to accompany the construction with a spacetime diagram or else consult Figure 1 of (Malament, 1996). The covariance properties of our projections are a consequence of the corresponding covariance properties of the effects and the spectral theorem. Let $\Delta \in \mathcal{F}(S)$ be a bounded spatial set and $a \in M$ tangent to $S$ such that $\Delta$ and $\Delta+a$ are disjoint and such that for all future directed timelike $a_{1}$ and all sufficiently small (in modulus) $t, \Delta$ and $\Delta+a+t a_{1}$ are spacelike separated. Due to the localization condition (2') one has

$$
\forall \varphi \in \mathcal{H} \quad(\|\varphi\|=1): \quad\left\langle\varphi \mid E_{\Delta} \varphi\right\rangle=1 \Longrightarrow\left\langle\varphi \mid E_{\Delta+a} \varphi\right\rangle=0 .
$$

This is equivalent to

$$
P_{\Delta}^{(1)}\left(I-P_{\Delta+a}^{(0)}\right)=0 .
$$

Applying translation covariance and locality, one obtains for sufficiently small $t$ :

$$
\left[E_{\Delta}, U\left(t a_{1}\right) E_{\Delta+a} U\left(t a_{1}\right)^{*}\right]=\left[E_{\Delta}, E_{\Delta+a+t a_{1}}\right]=0,
$$

and thus also (by virtue of the spectral theorem)

$$
\left[P_{\Delta}^{(1)}, U\left(t a_{1}\right)\left(I-P_{\Delta+a}^{(0)}\right) U\left(t a_{1}\right)^{*}\right]=\left[P_{\Delta}^{(1)}, I-P_{\Delta+a+t a_{1}}^{(0)}\right]=0 .
$$

Now apply Lemma 1 [taking $V(t)=U\left(t a_{1}\right), E_{1}=E_{\Delta}, E_{2}=I-P_{\Delta+a}^{(0)}$ ] - using the spectrum condition and (i), (ii) - to conclude that for all future directed timelike $a_{1}$, and all $t, P_{\Delta}^{(1)} U\left(t a_{1}\right)\left(I-P_{\Delta+a}^{(0)}\right) U\left(t a_{1}\right)^{*}=0$, and therefore

$$
P_{\Delta}^{(1)}\left(I-P_{\Delta+a+t a_{1}}^{(0)}\right)=0 .
$$


Next let $a_{2}$ be any future directed timelike unit vector. For sufficiently large $t_{2}>0$, the set $\Delta+t_{2} a_{2}$ is to the timelike future of $\Delta+a$. Then one can find $t_{2}>0$ and $\varepsilon>0$ such that $\Delta+\left(t_{2}+t\right) a_{2}$ is to the timelike future of $\Delta+a$ for all $t$ with $|t|<\varepsilon$. Hence if $|t|<\varepsilon$, there is a future directed timelike unit vector $a_{1}$ and a number $t_{1}$ such that $\Delta+\left(t_{2}+t\right) a_{2}=\Delta+a+t_{1} a_{1}$. Thus, by (iii), if $|t|<\varepsilon$, then $P_{\Delta}^{(1)}\left(I-P_{\Delta+\left(t_{2}+t\right) a_{2}}^{(0)}\right)=0$, or equivalently, by translation

covariance: $P_{\Delta}^{(1)} U\left(t a_{2}\right)\left(I-P_{\Delta+t_{2} a_{2}}^{(0)}\right) U\left(t a_{2}\right)^{*}=0$. Invoking Lemma 1again [with $\left.E_{1}=P_{\Delta}^{(1)}, E_{2}=I-P_{\Delta+t_{2} a_{2}}^{(0)}\right]$, one obtains

$$
P_{\Delta}^{(1)} U\left(t a_{2}\right)\left(I-P_{\Delta+t_{2} a_{2}}^{(0)}\right) U\left(t a_{2}\right)^{*}=0
$$

and hence (by translation covariance)

$$
P_{\Delta}^{(1)} U\left(\left(t+t_{2}\right) a_{2}\right)\left(I-P_{\Delta}^{(0)}\right) U\left(\left(t+t_{2}\right) a_{2}\right)^{*}=0
$$

for all $t$. Choosing $t=-t_{2}$, one concludes that $P_{\Delta}^{(1)}\left(I-P_{\Delta}^{(0)}\right)=0$, and so $P_{\Delta}^{(1)} \leq P_{\Delta}^{(0)}$, that is,

$$
P_{\Delta}^{(1)}=0
$$

\section{References}

[1] Schlieder, S. (1971). Zum kausalen Verhalten eines relativistischen quantenmechanischen Systems. In: Quanten und Felder, W. Heisenberg zum 70. Geburtstag. Ed. H.P. Dürr, Vieweg, pp. 145-160.

[2] Hegerfeldt, G.C. (1998). Instantaneous Spreading and Einstein Causality in Quantum Theory. Annalen der Physik 7, 716-725.

[3] Schweber, S.S. (1961). An Introduction to Relativistic Quantum Field Theory. Harper \& Row, New York.

[4] Greiner, W. (1994). Relativistic Quantum Mechanics. Springer-Verlag, Berlin.

[5] Wightman, A.S. (1962). On the Localizability of Quantum Mechanical Systems. Reviews of Modern Physics 34, 845-872.

[6] Ali, S.T. (1985). Stochastic Localization, Quantum Mechanics on Phase Space and Quantum Spacetime. La Rivista del Nuovo Cimento 8(11), 1128.

[7] Ali, S.T. (1998). Systems of Covariance in Relativistic Quantum Mechanics. International Journal of Theoretical Physics 37, 365-373.

[8] Greenwood, D.P., and Prugovečki, E. (1984). Stochastic microcausality in relativistic quantum mechanics. Found. Phys. 14, 883-906. 
[9] Brooke, J.A., and Schroeck, F.E. (1996). Localization of the photon on phase space. J. Math. Phys. 37, 5958-5986.

[10] Malament, D. (1996). In Defense of Dogma: Why There Cannot Be A Relativistic Quantum Mechanics of (Localizable) Particles. In: Perspectives on Quantum Reality, ed. R. Clifton, Kluwer Academic Publishers, Dordrecht.

[11] Newton, T.D., and Wigner, E.P. (1949). Localized States for Elementary Systems. Reviews of. Modern Physics 21, 400-406.

[12] Castrigiano, D.P.L., and Mutze, U. (1982). Covariant description of particle position. Journal of Mathematical Physics 26, 3499-3505.

[13] Borchers, H.J. (1967). A Remark on a Theorem of B. Misra. Communications in Mathematical Physics 4, 315-323.

[14] Giannitrapani, R. (1998). Quantum Coordinates of an Event in Local Quantum Physics. Journal of Mathematical Physics 39, 5180-5182.

[15] Schlieder, S. (1968). Einige Bemerkungen zur Zustandsänderung von relativistischen quantenmechanischen Systemen durch Messungen und zur Lokalitätsforderung. Communications in Mathematical Physics 7, 305-331.

[16] Lüders, G. (1951). Über die Zustandsänderung durch den Messprozess. Annalen der Physik (6.F.) 8, 322-328.

[17] Busch, P. and Singh, J. (1998). Lüders theorem for unsharp quantum measurements. Physics Letters A 249, 10-12.

[18] Busch, P., Grabowski, M. and Lahti, P. (1995). Operational Quantum Physics. Springer-Verlag, Berlin, 2nd Corrected Printing 1997.

[19] de Muynck, W.M. (1984). A quantum mechanical theory of local observables and local operations. Foundations of Physics 14, 199-253.

[20] Busch, P. (1999). To be published.

[21] Mittelstaedt, P. (1998). Can EPR-correlations be used for the transmission of superluminal signals? Annalen der Physik 7, 710-715.

[22] Butterfield, J. and Fleming, G. (1999). Strange Positions. In: From Physics to Philosophy. Eds. J. Butterfield, C. Pagonis, Cambridge University Press, to appear.

[23] Brooke, J.A., and Busch, P. (1999). In preparation. 